NBER WORKING PAPER SERIES

\title{
HETEROGENEOUS ACTIONS, BELIEFS, CONSTRAINTS AND RISK TOLERANCE DURING THE COVID-19 PANDEMIC
}

\author{
Ying Fan \\ A. Yeşim Orhun \\ Dana Turjeman \\ Working Paper 27211 \\ http://www.nber.org/papers/w27211 \\ NATIONAL BUREAU OF ECONOMIC RESEARCH \\ 1050 Massachusetts Avenue \\ Cambridge, MA 02138 \\ May 2020
}

Authors are listed alphabetically. We thank SafeGraph Inc. for data access, Max Resnick and Helen Wang for excellent research assistance and Lillian Chen for survey management. The University of Michigan IRB approved this study (HUM00148129, HUM00180582, and HUM00180640). The views expressed herein are those of the authors and do not necessarily reflect the views of the National Bureau of Economic Research.

NBER working papers are circulated for discussion and comment purposes. They have not been peer-reviewed or been subject to the review by the NBER Board of Directors that accompanies official NBER publications.

(C) 2020 by Ying Fan, A. Yeşim Orhun, and Dana Turjeman. All rights reserved. Short sections of text, not to exceed two paragraphs, may be quoted without explicit permission provided that full credit, including $\odot$ notice, is given to the source. 
Heterogeneous Actions, Beliefs, Constraints and Risk Tolerance During the COVID-19 Pandemic Ying Fan, A. Yeşim Orhun, and Dana Turjeman

NBER Working Paper No. 27211

May 2020

JEL No. D81,D84,D91,H41,I12,I18

\section{ABSTRACT}

During a pandemic, an individual's choices can determine outcomes not only for the individual but also for the entire community. Beliefs, constraints and preferences may shape behavior. This paper documents demographic differences in behaviors, beliefs, constraints and risk preferences across gender, income and political affiliation lines during the new coronavirus disease (COVID-19) pandemic. Our main analyses are based on data from an original nationally representative survey covering 5,500 adult respondents in the U.S. We find substantial gaps in behaviors and beliefs across gender, income and partisanship lines; in constraints across income levels; and in risk tolerance among men and women. Based on location data from a large sample of smartphones, we also document significant differences in mobility across demographics, which are consistent with our findings based on the survey data.

Ying Fan

Department of Economics University of Michigan

611 Tappan Street

Ann Arbor, MI 48109

and NBER

yingfan@umich.edu

A. Yeşim Orhun

Ross School of Business

University of Michigan

701 Tappan Street

Ann Arbor, MI 48109

aorhun@umich.edu
Dana Turjeman

Ross School of Business

University of Michigan

701 Tappan Street

Ann Arbor, MI 48109

turji@umich.edu 


\section{Introduction}

Individual behavioral responses during the outbreak of a highly contagious disease, such as the COVID-19 pandemic, contribute significantly to public health outcomes. Economic theory suggests that individual choices are shaped by beliefs, constraints, and preferences. Therefore, it is important to understand differences in these factors to coordinate an effective response.

We document substantial differences in behaviors, beliefs, constraints, and risk tolerance regarding the COVID-19 pandemic across gender, income, and political affiliation lines. Our analyses rely primarily on an original and nationally representative survey conducted weekly between April 1 and April 29, 2020 covering 5,500 individuals. We also use geo-location data from a large sample of smartphones provided by SafeGraph to examine differences in mobility responses.

We first document systematic differences along gender, income, and political affiliation lines in the extent to which Americans curtail their mobility outside the home. Using the geo-location data, we show that as the pandemic progresses, U.S. counties with higher shares of women and counties with lower poverty rates reduce both non-work and work trips more than counties in the same state that are otherwise similar in their local infection risks as well as their demographics and socio-economic metrics. Counties with higher shares of Democrats respond more in reducing non-work trips but less in limiting work trips.

Using survey data, we study a wide variety of social distancing choices that may contribute to these mobility differences, as well as other precautionary behaviors. Moreover, the survey allows us to examine heterogeneity not only in actions, but also in preferences, constraints, and beliefs. We find significant heterogeneity in behaviors and the factors that may shape them among people who are otherwise similar and live in communities that face similar COVID-19 risks.

Compared to their respective counterparts, Democrats and women are more likely to limit socializing with members outside of their household and take other health precautions (e.g., wiping down groceries, washing hands, and wearing masks), whereas low-income households are less likely to do so. Low-income households are also less likely to work from home and to avoid large gatherings and public transportation.

We also find heterogeneity in beliefs across these demographic lines. Women and Democrats are more pessimistic about their own health outcomes and/or chances of being infected, expect 
more deaths in the U.S., and believe more strongly in the effectiveness of state restrictions. They are also more worried about the health and economic well-being of themselves and those around them. In contrast, while low-income respondents worry more about their own economic well-being than high-income respondents do, they hold more optimistic beliefs about deaths in the U.S. and believe less in the effectiveness of state restrictions.

Finally, we find that female respondents and Democrats are less willing than their respective counterparts to tolerate risk, and that respondents in low-income households are more likely to be constrained in their ability to self-isolate and to work from home. After presenting these results, we discuss the relationship between our survey results and the patterns we document in geographic mobility differences. We also discuss the association between differences in individuals' behavioral responses and differences in their beliefs about the COVID-19 pandemic.

Our paper provides insights about several demographic differences regarding a wide span of behaviors and beliefs, while also examining differences in constraints and risk tolerance. In addition to documenting partisan differences, we study gaps along gender and income lines, which prove to be significant. The behaviors we study include work and non-work mobility, as well as social distancing choices and other precautionary behaviors, providing a comprehensive picture of demographic differences in response to the COVID-19 pandemic. Because demographic gaps in beliefs may not - and indeed do not, according to our results - consistently go in the same direction, we examine several types of beliefs that may influence individuals' decisions: beliefs regarding systemic risk, individual risks of infection and health outcome conditional on infection, and effectiveness of personal and policy efforts. Furthermore, we explore differences in risk tolerance and constraints, two factors that may also shape behaviors. We document income gaps in constraints and gender gaps in risk tolerance. While more research is needed to separately quantify the effects of beliefs, constraints and preferences on decision-making in this pandemic, our results suggest important differences across demographic dimensions in the extent to which each of these factors shapes individual behaviors.

Across gender lines, our results indicate significant differences in behaviors, beliefs and risk tolerance. A priori, it is unclear how women and men may differ in their work-related mobility responses and their willingness to take discretionary precautions. For example, women may be more likely to have jobs that cannot be done at home, but those who can afford it may also be more 
likely to stop working to take over childcare and homeschooling (e.g., Adams-Prassl et al. 2020). Similarly, while women tend to have more friends and derive more support from them (e.g., Walen and Lachman 2000), they may also be more willing to limit socialization and take other precautions because in certain contexts they tend to be more risk averse and/or more likely to make sacrifices for the greater good (e.g., Andreoni and Vesterlund 2001, Croson and Gneezy 2009, Shurchkov and Eckel 2018). In net, we find that women are more likely than men to limit work trips, less likely to socialize with friends and extended family and more likely to engage in precautionary health behaviors. The gender differences in beliefs and risk tolerance we document may contribute to these findings. Evidence suggests that women's risk aversion may lead them to make more conservative choices (e.g., Jianakoplos and Bernasek 1998, Barber and Odean 2001). To the extent that the risk preference difference contributes to gender differences in actions, our results suggest that women's lower risk tolerance may also be socially beneficial, as personal preventive actions are likely to have positive health spillovers on others.

Across the political divide, we see substantial differences in behaviors and beliefs. Several concurrent papers document partisan differences in expectations of U.S. deaths and beliefs regarding the effectiveness of social distancing (Allcott et al. 2020, Kushner Gadarian et al. 2020), as well as differences in limiting visits to locations of interest outside of home (e.g., restaurants, retail) before most states introduced mobility restrictions (Allcott et al. 2020, Andersen 2020, Painter and Qiu 2020). By documenting the differences in beliefs about the COVID-19 pandemic, we add to the literature on partisan perception differences regarding objective outcomes (e.g., Garrett and Lange 1989, Gaines et al. 2007). Evidence on how perception differences along partisan lines translate into behavioral differences is mixed (e.g., Gerber and Huber 2009, McGrath et al. 2017). Testing whether differences in beliefs drive differences in actions is beyond the scope of this paper. Nonetheless, it is interesting that controlling for belief differences almost completely attenuates the behavior differences we document across the political affiliation divide, and controlling for differences in news consumption completely attenuates the gaps in beliefs. These results suggest that consistent and accurate information could play an important role in combating COVID-19.

Our results along income lines also point to important disparities that policy makers should consider. Low-income individuals are over-represented in jobs that cannot be done from home; and consequently, they are more at risk of exposure and job loss (e.g., Adams-Prassl et al. 2020). 
At the same time, low-income households are more likely to experience financial strain and have lower access to credit, health care and health insurance (e.g., Berchick et al. 2019). This paper documents that low-income individuals are less likely to reduce mobility and are more constrained in their ability to work from home and in self-isolate. These findings imply an additional disadvantage that they face during the pandemic. Public policies may need to pay special attention to easing the constraints on those most severely affected by the pandemic.

\section{Observed Mobility Differences: SafeGraph Data Results}

In this section, we present empirical patterns from the SafeGraph data as a starting point for our investigation. SafeGraph is a data company that aggregates anonymized location data from numerous applications to provide insights about physical places (www.safegraph.com). Using geolocation data obtained from the activity of more than 35 million smartphones, SafeGraph provides an aggregated dataset (Social Distancing Metrics) to researchers interested in exploring COVID19-related topics. To enhance privacy, SafeGraph excludes a census block group if it has fewer than five devices observed in a month. For each census block on a given day, Social Distancing Metrics data report the number of devices exhibiting full-time work behavior (spending more than 6 hours between 8 a.m. and 6 p.m. in one location outside home), part-time work behavior (spending more than 3 but less than 6 hours between 8 a.m. and 6 p.m. in one location outside home), and the number of devices that stay home all day. ${ }^{1}$

We aggregate these data from their original census block group level to the county level and augment them with county election results from the 2016 presidential election (from MIT Election Data and Science Lab (2018)) as well as county COVID-19 case and death numbers, county demographics data on population density, age, education, race, poverty and employment, and rural-urban descriptors (from Killeen et al. (2020)). Appendix A contains further details on data construction.

We examine how the shares of devices that left home (for any reason), the shares of devices that left home for full-time work, for part-time work and for non-work reasons vary with county

\footnotetext{
${ }^{1}$ SafeGraph determines the home of a device by its common nighttime location over a 6 -week period.
} 
demographics between February 1 and April 24 of 2020. Our main specification is as follows:

$$
s_{c t}=D_{c} \beta_{w(t)}+Z_{c t} \gamma_{w(t)}+\zeta_{c}+\eta_{s(c), t}+\varepsilon_{c t},
$$

where $s_{c t}$ is the share of devices in county $c$ on day $t$ exhibiting a particular behavior and $D_{c}$ is a vector of county descriptors of interest: percent female, percent voting for Clinton in the 2016 election (termed "Democrats" in the discussion of results), and percent living under the poverty line. The coefficients of interest, $\beta_{w(t)}$, are allowed to vary by week $w(t)$. The control variables $Z_{c t}$ are also allowed to vary weekly in their influence. They include demographic and socioeconomic controls, and variables to capture differences in COVID-19 risks across counties. ${ }^{2}$ We include county fixed-effects $\left(\zeta_{c}\right)$ to control for cross-sectional differences and state-date fixed effects $\left(\eta_{s(c), t}\right)$ to control for state-wide changes in social-distancing policies. Because each observation is a share in a county, we weight observations by the total number of devices in each county. We also cluster errors at the county level.

The dependent variables are computed using the total number of devices in the sample as the denominator and the number of devices exhibiting certain behaviors as the numerators. SafeGraph reports a sampling bias in favor of detecting devices that are moving (SafeGraph 2020). We adjust the share of devices ever leaving home by designating all devices that were inactive on a specific day and in the sample in that month as "at-home." 3

Each column of Figure 1 corresponds to a share of devices exhibiting a certain behavior. Each row corresponds to a demographic variable. The coefficients are estimated relative to the first week in our sample. The pattern over time is consistent across columns: while differences are nonexistent or small during February, in cases where differences emerge, plots start to fan out from the zero-line in early March and stabilize in April. Recall that the first confirmed case in the U.S. was reported on January 21. States that eventually put restrictions in place did so primarily in the last week of March or first week of April (see Figure 2 for a timeline of various state interventions).

\footnotetext{
${ }^{2}$ These control variables are population shares of whites, blacks, and Asians, percent of adults with a bachelor's degree, percent of adults without a high-school degree, unemployment rate, and the employed share of the county population, as well as logarithm of one plus the number of COVID-19 cases, logarithm of one plus the number of COVID-19 deaths, logarithm of population density, population share over the age of 65, degree of urbanization, and a binary variable indicating whether the county is in a metropolitan area.

${ }^{3}$ Supplemental Appendix SA details this sampling bias and shows the robustness of our results to omitting this adjustment, to using several different adjustment approaches, and to including varying sets of control variables.
} 
As the pandemic progresses, the estimates indicate that compared to Republicans, Democrats respond more in reducing non-work trips, but less in limiting work-related trips. U.S. counties with high poverty rates become less likely to reduce work and non-work trips than counties with higher earnings. In contrast, counties with a higher share of women respond more strongly in limiting non-work and work trips than counties with lower shares of women. The demographic differences in work travel could be related to differences in the representation of females, low-income workers, and Democrats in essential jobs and in workplaces allowed or required to stay open. ${ }^{4}$ Non-work trips may include both necessities (e.g., grocery shopping) and discretionary trips (e.g., visiting friends and extended family). Next, we use our survey data to study different types of social distancing choices that may contribute to these mobility differences. In Section 4, we discuss these mobility results in light of what we learn from the survey.

\section{Actions, Beliefs, Constraints and Risk Tolerance: Survey Results}

This section examines how different types of social distancing choices and other preventive choices, as well as potential drivers of actions (i.e., beliefs, constraints, and risk tolerance) vary across partisan, gender, and income lines. To this end, we conducted a survey in five weekly waves during April 2020. Our sample comprises 5,500 respondents and is nationally representative in geography, age, gender and ethnicity (see Appendix B for survey details).

To examine heterogeneity in actions, beliefs, constraints and risk tolerance, we use the following regression equation:

$$
Y_{i}^{\tau}=D_{i} \alpha+X_{i \tau} \lambda+\xi_{s(i) \tau}+\varepsilon_{i \tau}
$$

where $Y_{i}^{\tau}$ is an outcome variable of interest, i.e., an action, belief, constraint, or preference of respondent $i$ at time $\tau$. We explain these dependent variables as we discuss our results. The vector $D_{i}$ represents the demographic variables of interest: respondent $i$ 's political affiliation (Democrat, Republican, or Independent), gender (female or male), and annual household income (less than $\$ 30,000$, between $\$ 30,000$ and $\$ 74,999$, or above $\$ 75,000)$. The control variables $X_{i \tau}$ include the

\footnotetext{
${ }^{4}$ For example, Sperling (2020) and Ingraham (2020) note that many essential workers tend to have lower incomes. Delaporte and Peña (2020) show that higher-income individuals are more likely to be able to work from home due to the nature of their jobs. Similarly, Adams-Prassl et al. (2020) note that lower-income individuals are more likely to work in occupations in which only a small share of tasks can be done from home. Studying mobility in New York City, Coven and Gupta (2020) show that low-income residents are less likely to stay at home.
} 
population density of the respondent's zip code, the number of positive COVID-19 cases and deaths in the respondent's county at the time of the survey, and demographic variables such as age, race, educational level, and current employment status. Finally, we include state-time fixed effects, $\xi_{s(i) \tau}$, where subscripts refer to state $s(i)$ at time $\tau$, to capture systematic differences across states at the time of each survey wave.

\subsection{Heterogeneous Actions}

In the survey, we ask "Which of the following changes have you personally made to protect yourself from the coronavirus infection?" for a set of actions: (1) self-isolate at home and avoid all public places; (2) avoid public transportation and large gatherings; (3) work from home; (4) do not meet friends or extended family; (5) wash hands more often; (6) wipe down groceries after bringing them home; (7) wear a mask when out and about; and (8) wear gloves when going shopping. ${ }^{5}$ These questions fall into two broad groups: social distancing decisions that may contribute to the mobility differences documented in Section 2 (items (1) - (4); results presented in the left panel of Figure 3), and other health precautions individuals may take that are difficult to glean from any observational data (items (5) - (8); results presented in the right panel of Figure 3).

Overall, our results suggest that women and Democrats are taking more precautions than their counterparts and that low-income individuals are less likely to do so than higher-income ones. The results in the left panel of Figure 3 document that low-income respondents are less likely to work from home or avoid large gatherings, and that Democrats and women are more likely to avoid large gatherings than Republicans and men. The decision to stop seeing friends and extended family may be the most discretionary of all actions contributing to mobility. Democrats and women are more likely to limit socialization than Republicans and men, while low-income respondents are less likely to do so than high-income respondents. The gender difference in the propensity to stop socializing with friends and extended family is the largest across all actions studied. The right panel of Figure 3 shows that low-income individuals are less likely to take other health precautions than higher-income respondents, whereas Democrats and women are more likely to do so than

\footnotetext{
${ }^{5}$ The survey also asked whether respondents canceled travel plans and an open-ended question about any additional precautions they took. A very low proportion of respondents took other precautions. Travel cancellation is heavily influenced by having travel plans to begin with. Nonetheless, using the same regression (2), we find that low-income individuals are less likely to cancel travel plans. There are no gender or partisan differences in cancelling travel plans.
} 
their counterparts. As Supplementary Appendix SB reports, these results are robust to controlling for respondents' personal experience (i.e., whether the virus has affected a respondent's health or the health of a family member or a close friend) and pre-existing health conditions (diabetes, high blood pressure, chronic pulmonary disease, etc.). Next, we examine heterogeneity in factors that may contribute to differences in these decisions.

\subsection{Heterogeneous Beliefs and Worries}

The survey asked respondents how worried they feel for their own health, and the health of their partner, kids, extended family, members of their community, and the whole U.S. It also asked how worried they feel for the economic well-being of the same groups of people. The survey also elicited three types of beliefs: (1) Individuals' predictions on their own health risks: (1.1) chance of becoming infected in the next three months assuming that the social distancing policies and their personal protection efforts stay the same; (1.2) chance of having no or mild symptoms should they get infected. (2) Their predictions on systemic health risks: the number of U.S. deaths by July 1, 2020 assuming the state policies remain the same. (3) Their beliefs regarding the effectiveness of state policies and preventive actions: (3.1) how their infection chance would change if their state had not introduced social distancing measures, assuming their personal efforts stay the same; (3.2) how their infection chance would change if they had not taken preventive actions, assuming that the state policies stay the same; and (3.3) their prediction on the number of U.S. deaths by July 1, 2020 if there were no social distancing measures.

Panels (a) and (b) of Figure 3 present heterogeneity in predictions regarding own or systemic health risks and the effectiveness of state policies and personal precautions, respectively. Democrats (and to a lesser extent, Independents) are generally more pessimistic about health outcomes and believe more in the effectiveness of policies and preventive actions than Republicans. ${ }^{6}$ Women are more pessimistic than men about their health outcomes should they get infected and are stronger believers than men in the effectiveness of state policies and of their own preventive actions. Lowincome respondents are more optimistic about the number of deaths they expect in the U.S. and do not believe in the effectiveness of personal measures or state policies as much as higher-income

\footnotetext{
${ }^{6}$ Beliefs regarding effectiveness of own actions could be driven partially by differences in the propensity to take precautions.
} 
households do.

In addition to gaps in beliefs, we also see that women and Democrats worry more than their counterparts about health and economic well-being (Figure 4, panels (c) and (d)), ranging from their own health or economic well-being to their partner's, local community's, and the whole nation's. The difference between women and men is the largest for worries about their children's health. How much respondents worry about their own health or the health of others does not seem to vary based on household income, but low-income respondents worry more about their own economic well-being than high-income ones do.

These results are robust to controlling for respondents' personal experience and pre-existing health conditions (see Supplemental Appendix SB). Moreover, these belief differences line up with the differences in actions we have documented. We discuss this association in Section 4, where we also explore heterogeneity in news consumption.

\subsection{Heterogeneous Constraints}

To understand the constraints in people's ability to take preventive actions and how such constraints vary demographically, we asked people who said they had not taken an action to indicate the reason that best describes why. The response options fall into "constrained" and "unconstrained" categories. For example, if a respondent does not work from home, we consider the reason to be in the "constrained" category if her answer indicates that her work cannot be done from home or her employer did not give her the option. However, if her answer is "I could perhaps work from home, but it's better not to / I don’t like to," we consider her choice to be unconstrained.

Overall, our results suggest that constraints play some role in people's ability to take certain preventive actions. We find that a non-negligible proportion of people indicate constraints as the main reason for not taking certain actions. For example, $19.3 \%$ of respondents who continued to work outside cited constraints as the main reason for not working from home. Similarly, $12.4 \%$ of respondents who did not avoid all public places or self-isolate at home indicated that they did not do so because of constraints. ${ }^{7}$ We also find heterogeneity in constraints across demographics. Figure 5 shows that the extent to which constraints are reported as the main reason for not isolating

\footnotetext{
${ }^{7}$ In computing these shares and constructing the sample for the estimation in this section, we drop respondents whose answers indicate that the action is not applicable in their case.
} 
and not working from home is much higher for low-income respondents. All other estimates are statistically insignificant, indicating that for those who do not take these preventive actions, there is no systematic partisan or gender differences in their abilities to do so.

\subsection{Heterogeneous Risk Preference}

To explore systematic differences in risk tolerance, we add to our April 22 and 29 surveys the question "Thinking about yourself, in general, how willing or unwilling are you to take risks?" (ratings ranged from 0 "completely unwilling to take risks" to 10 "very willing to take risks"). This subjective self-assessment has been shown to be correlated with risk-taking behaviors in field and laboratory experiments and is used to measure risk preferences across the world (e.g., Vieider et al. 2015, Falk et al. 2018).

We find women to be less willing than men to take risks even after controlling for socio-economic and demographic differences such as employment status and educational level. The average score in the sample is 5.45 with a standard deviation of 2.65. Examining heterogeneity across demographics, we find a substantial gender gap: women are much less risk tolerant than men $(\alpha=0.88$, s.e. $=0.12)$. Republicans are, on average, more willing take risks, but the partisan gaps are smaller than the gender gap: the estimated differences are 0.47 points $($ s.e. $=0.14)$ for Democrats vs. Republicans and 0.45 points (s.e. $=0.15)$ for Independents vs. Republicans.

\section{Discussion}

In this section, we discuss the relationships among the various results we have documented thus far. We first highlight the relationship between mobility patterns documented in Section 2 and our survey results presented in Section 3. Recall that our analysis of the SafeGraph data suggests that people who live in counties with higher shares of Democrats and women and lower shares of poverty respond more strongly to the pandemic in limiting their non-work trips than their counterparts. These non-work trips may include discretionary outings and necessary trips like grocery shopping. Our survey confirms that Democrats, women and higher-income people are less likely to meet friends and extended family, suggesting that differences in non-work trip patterns are not driven solely by differences in necessities. 
At the same time, the survey also highlights that differences in necessities do seem to matter. When asked the main reasons they did not work from home or avoid public places and self-isolate, lower-income individuals are more likely to cite constraints than higher-income ones. This result suggests that the larger reduction in work trips in counties with lower poverty shares is likely to be driven by differences in necessities and in demographics of essential workers, not just by personal choice.

In addition to explaining mobility differences, the survey contributes to our understanding of other precautions individuals may take. Overall, we see that the propensity to limit discretionary mobility and the likelihood of taking other health precautions go together.

Furthermore, looking across the demographic differences in actions and beliefs, we see that groups that engage more in preventive actions are also more worried about the health and economic impact of the pandemic, are less optimistic about their infection risks and health outcomes, and are stronger believers in the effectiveness of state policies and personal preventive actions. In sum, heterogeneity in actions and heterogeneity in beliefs seem to be consistent with each other. To what extent does controlling for heterogeneity in beliefs attenuate heterogeneity in actions?

To answer this question, we repeat our action regressions, now including elicited beliefs as control variables (see Appendix $\mathrm{C}$ for more details and results). Once we account for heterogeneity in beliefs, the behavioral differences between Democrats and Republicans in their propensity to physically distance themselves from others or to take other preventive actions are largely attenuated (except for the differences in wearing masks). Though this exercise is not meant to identify a causal impact of beliefs on actions, ${ }^{8}$ this result points to a strong association between differences in beliefs and differences in actions across partisan lines.

After we control for belief differences, women remain more likely to stop seeing friends and extended family, wear masks, and wash hands more often. Similarly, the findings that low-income respondents are less likely to work from home or stop seeing friends and extended family remain significant. These results suggest that additional factors are important in understanding these behavioral differences across gender and income. This paper highlights constraints and risk preferences as two examples of such factors.

\footnotetext{
${ }^{8}$ Beliefs may be endogenous in such regressions, for instance, due to motivated reasoning or due to a common unobservable shock to actions and beliefs.
} 
Recall that we find large differences in beliefs across individuals facing the same public health crisis. Such belief heterogeneity could be related to systematic differences in information sources. To examine this possibility, we asked survey respondents about the news sources they usually rely on and how much attention they pay to other information sources such as friends, their governor, the President and his administration, etc. Using these data, we repeat our belief regressions after adding controls for news sources that respondents find reliable and other information sources that respondents pay attention to (see Appendix D for more details on these control variables and estimation results). We find that variations in news and other information sources almost entirely attenuate belief differences across political affiliation. The attenuation is less pronounced for differences across gender or household income. While this result does not necessarily imply a causal effect of news consumption on beliefs, the asymmetry in the attenuation effect of these controls suggests a partisan difference in the type of information people consume - a fact that has been independently documented in other contexts. ${ }^{9}$ Because partisan differences in news consumption can lead to differences in behaviors, our results suggest the importance of disseminating consistent public messages about the COVID-19 pandemic for an effective public health response.

\section{Conclusion}

By documenting heterogeneity across demographics in people's actions, beliefs, constraints and risk preferences in the COVID-19 pandemic, this paper takes a step toward understanding how the pandemic affects people in different demographic groups and aims to inform targeted policy discussions as the economy opens up. For example, to the extent that beliefs shape actions, an effort to reach people of all ideologies with a consistent message may help to unify the public response to the pandemic. We also find that a significant proportion of respondents cite constraints as the main reason for not working from home or self-isolating; moreover, this share is larger for lower-income households. Furthermore, we find that women are, on average, significantly less tolerant of risk. They also worry more about their own health and the health of those around them (in particular, their kids' health) and take more preventive actions. These differences should be considered in policy discussions, especially since early efforts to reopen the economy are likely to

\footnotetext{
${ }^{9}$ For example, Gentzkow and Shapiro (2011) and Bakshy et al. (2015) show partisan differences in news consumption.
} 
have a disproportionate impact on these demographic groups.

\section{References}

Adams-Prassl, Abi, Teodora Boneva, Marta Golin, and Christopher Rauh (2020), "Inequality in the impact of the coronavirus shock: Evidence from real time surveys." IZA Discussion Papers 13183.

Allcott, Hunt, Levi Boxell, Jacob Conway, Matthew Gentzkow, Michael Thaler, and David Y Yang (2020), "Polarization and public health: Partisan differences in social distancing during the coronavirus pandemic." NBER Working Paper 26946.

Andersen, Martin (2020), "Early evidence on social distancing in response to COVID-19 in the United States." SSRN Working Paper 3569368.

Andreoni, James and Lise Vesterlund (2001), "Which is the fair sex? Gender differences in altruism." The Quarterly Journal of Economics, 116, 293-312.

Bakshy, Eytan, Solomon Messing, and Lada A Adamic (2015), "Exposure to ideologically diverse news and opinion on facebook." Science, 348, 1130-1132.

Barber, Brad M and Terrance Odean (2001), "Boys will be boys: Gender, overconfidence, and common stock investment." The Quarterly Journal of Economics, 116, 261-292.

Berchick, Edward R., Jessica C. Barnett, and Rachel D. Upton (2019), "Health insurance coverage in the United States: 2018." Current population reports, U.S. Census Bureau.

Coppock, Alexander and Oliver A. McClellan (2019), "Validating the demographic, political, psychological, and experimental results obtained from a new source of online survey respondents." Research $\mathscr{E}$ Politics, $6,1-14$.

Coven, Joshua and Arpit Gupta (2020), "Disparities in mobility responses to COVID-19." working paper, New York University.

Croson, Rachel and Uri Gneezy (2009), "Gender differences in preferences." Journal of Economic Literature, $47,448-74$.

Delaporte, Isaure and Werner Peña (2020), "Working from home under COVID-19: Who is affected? Evidence from Latin American and Caribbean countries." GLO Discussion Paper 508.

Falk, Armin, Anke Becker, Thomas Dohmen, Benjamin Enke, David Huffman, and Uwe Sunde (2018), "Global evidence on economic preferences." The Quarterly Journal of Economics, 133, 1645-1692.

Gaines, Brian J, James H Kuklinski, Paul J Quirk, Buddy Peyton, and Jay Verkuilen (2007), "Same facts, different interpretations: Partisan motivation and opinion on Iraq." The Journal of Politics, 69, 957-974. 
Garrett, Geoffrey and Peter Lange (1989), "Government partisanship and economic performance: When and how does "who governs" matter?" The Journal of Politics, 51, 676-693.

Gentzkow, Matthew and Jesse M Shapiro (2011), "Ideological segregation online and offline." The Quarterly Journal of Economics, 126, 1799-1839.

Gerber, Alan S and Gregory A Huber (2009), "Partisanship and economic behavior: Do partisan differences in economic forecasts predict real economic behavior?" American Political Science Review, 103, 407-426.

Ingraham, Christopher (2020), "Analysis - Why many essential workers get paid so little, according to experts." Washington Post.

Jianakoplos, Nancy Ammon and Alexandra Bernasek (1998), "Are women more risk averse?" Economic Inquiry, 36, 620-630.

Killeen, Benjamin D, Jie Ying Wu, Kinjal Shah, Anna Zapaishchykova, Philipp Nikutta, Aniruddha Tamhane, Shreya Chakraborty, Jinchi Wei, Tiger Gao, Mareike Thies, et al. (2020), "A county-level dataset for informing the United States' response to COVID-19." arXiv preprint arXiv:2004.00756.

Kushner Gadarian, Shana, Sara Wallace Goodman, and Thomas B Pepinsky (2020), "Partisanship, health behavior, and policy attitudes in the early stages of the COVID-19 pandemic." SSRN Working Paper 3562796.

McGrath, Mary C et al. (2017), "Economic behavior and the partisan perceptual screen." Quarterly Journal of Political Science, 11, 363-83.

MIT Election Data and Science Lab (2018), "County presidential election returns 2000-2016."

Painter, Marcus and Tian Qiu (2020), "Political beliefs affect compliance with covid-19 social distancing orders." SSRN Working Paper 3569098.

SafeGraph (2020), "Safegraph's data analysis methodology."

Shurchkov, Olga and Catherine C Eckel (2018), Gender differences in behavioral traits and labor market outcomes. Oxford, UK: Oxford University Press.

Sperling, Gene B. (2020), "Opinion - Martin Luther King Jr. predicted this moment." The New York Times. Vieider, Ferdinand M, Thorsten Chmura, Tyler Fisher, Takao Kusakawa, Peter Martinsson, Frauke Mattison Thompson, and Adewara Sunday (2015), "Within-versus between-country differences in risk attitudes: Implications for cultural comparisons." Theory and Decision, 78, 209-218.

Walen, Heather R and Margie E Lachman (2000), "Social support and strain from partner, family, and friends: Costs and benefits for men and women in adulthood." Journal of Social and Personal Relationships, 17, 5-30. 


\section{Figures}

Figure 1: Shares of Devices Engaging in Different Types of Activity, SafeGraph Data

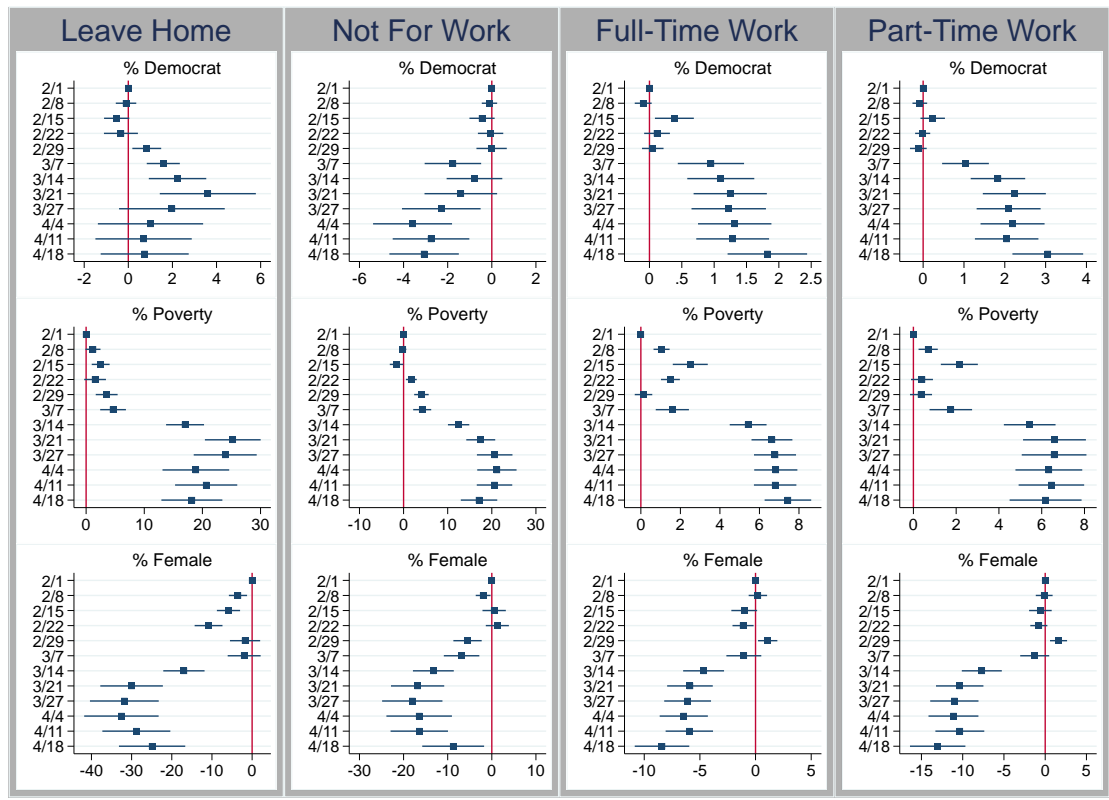

Notes: This figure plots the $\beta_{w(t)}$ estimates in equation (1) and the corresponding $95 \%$ confidence intervals. Each row plots the estimated coefficient for a specific demographic variable (\% Democrat, \% population below poverty line, and $\%$ female). The y-axis markers indicate the beginning date of the week for which the coefficients are reported.

Figure 2: Timing of Non-Pharmaceutical Interventions in the U.S.

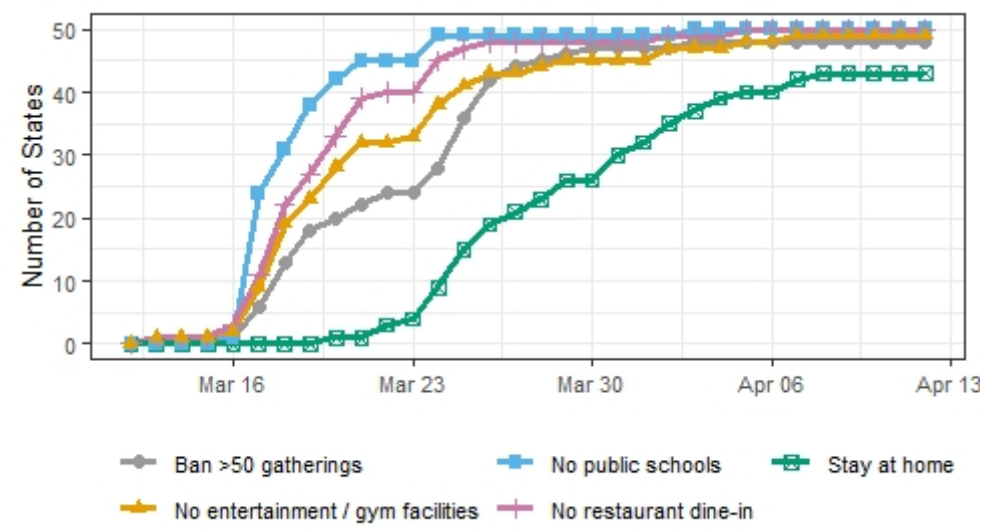

Notes: This figure plots the number of states introducing each type of intervention denoted in the legend. We exclude Alaska and Puerto Rico. Some states have not explicitly limited the size of gatherings, but such limits are implied by a stay-at-home order. Data: Killeen et al. (2020). 
Figure 3: How Actions Vary with Demographics, Survey Data

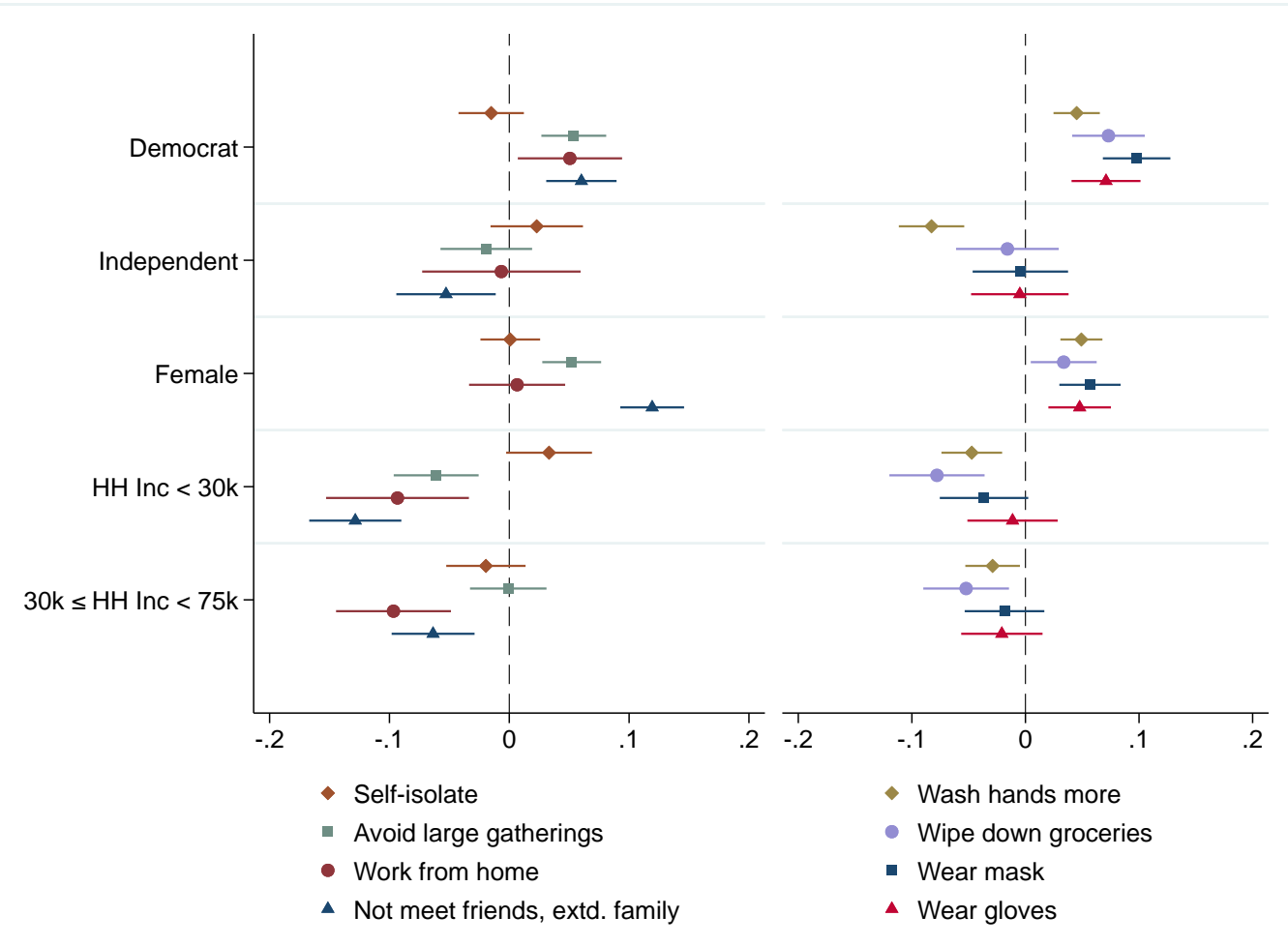

Notes: This figure plots the estimated coefficients $\alpha$ in equation (2) and the corresponding $95 \%$ confidence intervals. Each dependent variable is an indicator of whether the respondent took a certain action indicated in the legend. 
Figure 4: How Beliefs and Worries Vary with Demographics, Survey Data

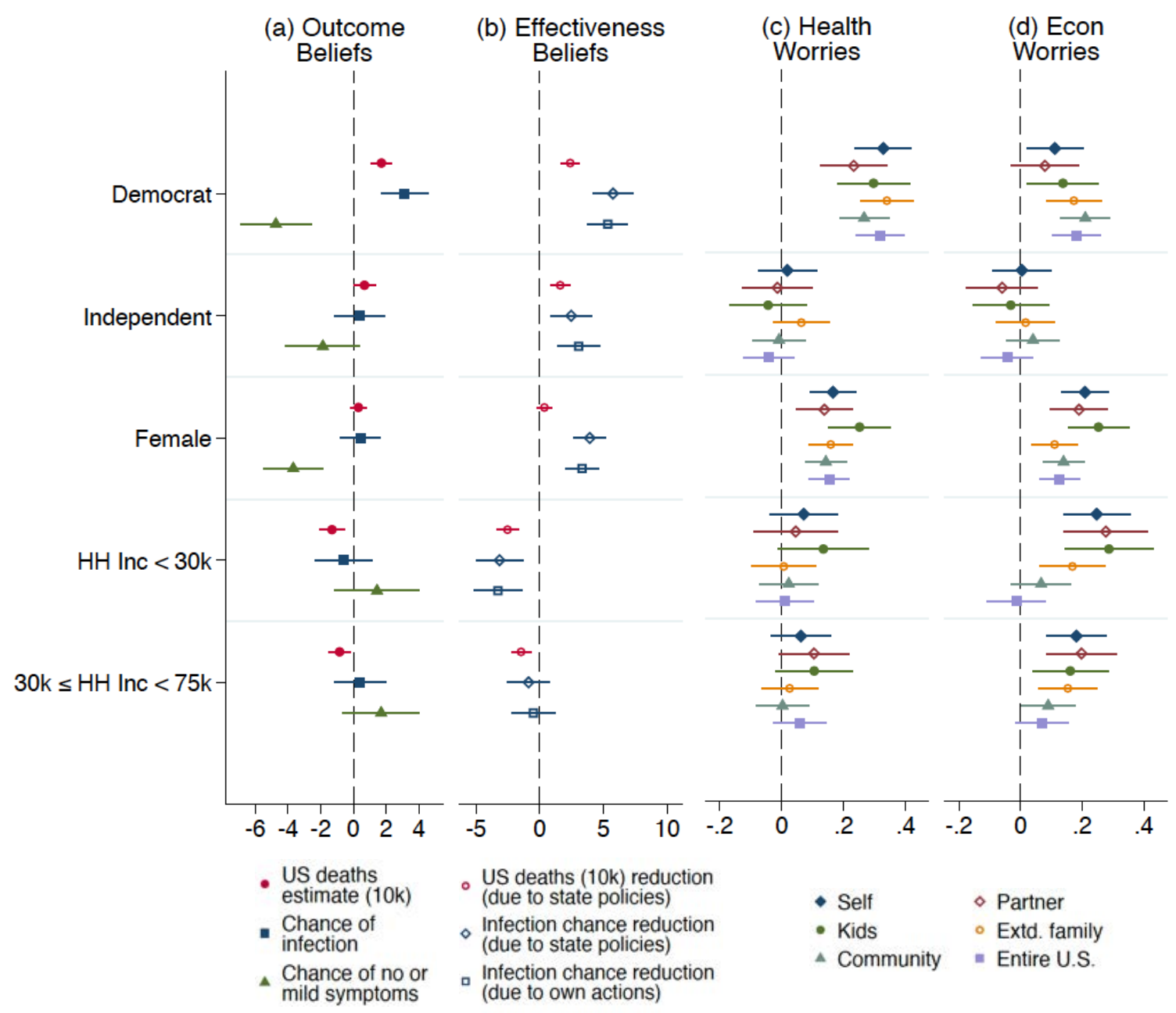

Notes: This figure plots the estimated coefficients $\alpha$ in equation (2) and the corresponding $95 \%$ confidence intervals. In panels (a) and (b), the dependent variables are elicited predictions indicated in the legend. In panels (c) and (d), the dependent variables are the extent to which respondents are worried about the well-being of the groups indicated in the legend. 
Figure 5: How Constraints as Main Reason for Not Taking an Action Vary with Demographics, Survey Data

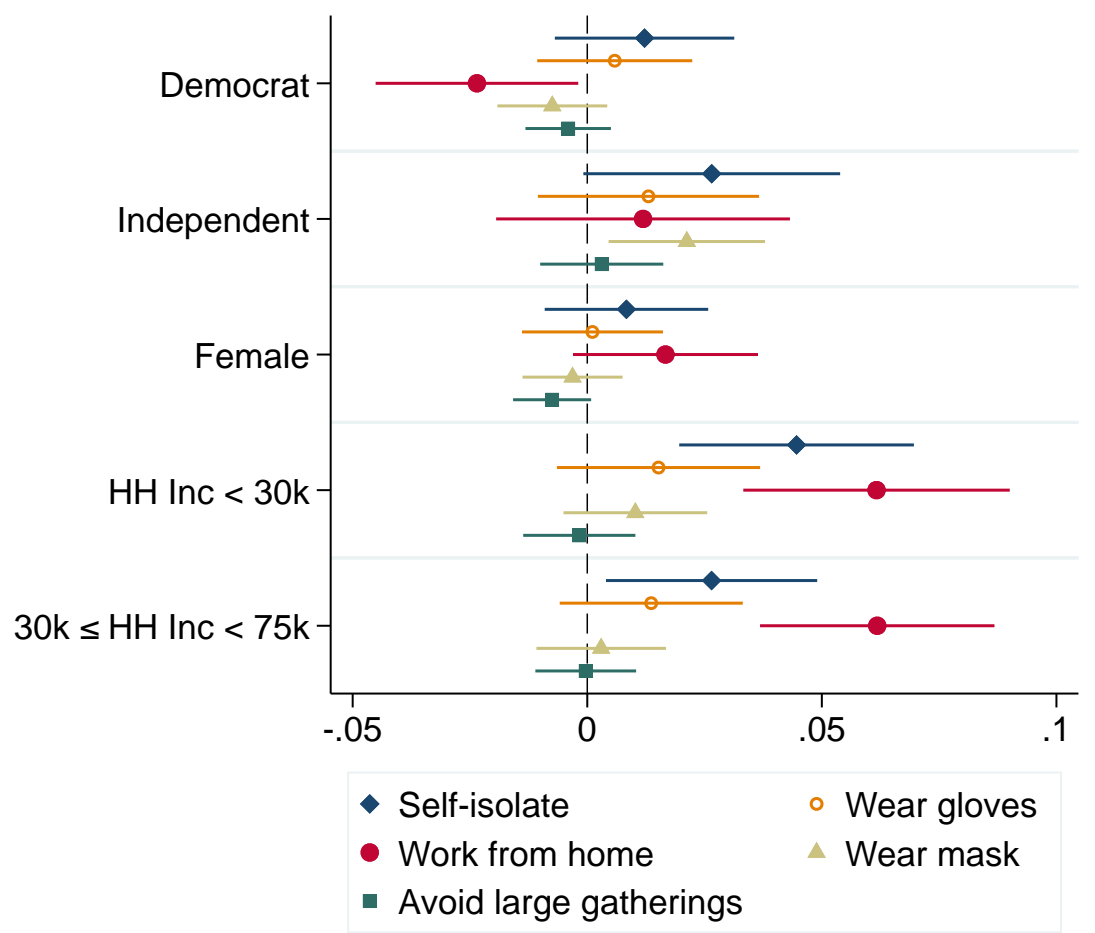

Notes: This figure plots the estimated coefficients $\alpha$ in equation (2) and the corresponding $95 \%$ confidence intervals. Dependent variables are indicators for whether a respondent cited constraints as the main reason for not taking an action indicated in the legend. 


\section{Appendices}

\section{A SafeGraph Data Details}

We aggregate the data from the Census Block Group to the county level and merge the countylevel demographic, voting, and COVID-19 cases and deaths datasets with the social distancing data. We exclude Alaska and territories of the U.S. Following SafeGraph notifications, we drop one date $(2 / 25 / 2020)$ known to cause a disproportional number of people to appear to be at home even if they were not.

The SafeGraph data are nationally representative in the three demographic aspects we study. Table A.1 shows that the national shares and sample shares are very close.

Table A.1: Representativeness of SafeGraph Data

\begin{tabular}{lcc}
\hline & National Shares & Sample Shares $^{a}$ \\
\hline Female Share & 0.520 & 0.521 \\
Democratic Share & 0.486 & 0.468 \\
Poverty Share & 0.128 & 0.128 \\
\hline${ }^{a}$ Average across counties weighted by the SafeGraph device count in each county.
\end{tabular}

\section{B Survey Details}

We repeated our survey across five weekly waves spanning April, 2020: April 1 (N=482), April 8 $(\mathrm{N}=1,974)$, April $15(\mathrm{~N}=1,027)$, April $22(\mathrm{~N}=988)$, and April $29(\mathrm{~N}=1,029)$. All surveys included questions on expectations and worries about how the pandemic may affect the health and economic well-being of themselves and others, policy attitudes, and news consumption patterns. Questions on self protective actions were introduced on April 8. Questions about constraints were introduced on April 15; the risk preference assessment was introduced on April 22. The survey is replicated in Supplemental Appendix SC.

We fielded the survey on Lucid (https://luc.id/theorem/) and aimed for a nationally representative population of adults (age 18 or over) living in the U.S. ${ }^{10}$ Our data are indeed representative of the U.S. adult population in terms of age, ethnicity, gender, political affiliation, income and

\footnotetext{
${ }^{10}$ An independent academic study, Coppock and McClellan (2019), examined the validity of Lucid in terms of respondent characteristics and treatment effect estimates and concluded that "subjects recruited from the Lucid platform constitute a sample that is suitable for evaluating many social scientific theories, and can serve as a drop-in replacement for many scholars currently conducting research on Mechanical Turk or other similar platforms."
} 
geographic regions (see Supplementary Appendix SC). The University of Michigan Institutional Review Board (IRB) reviewed the surveys and determined that they are exempt from ongoing review (HUM00148129, HUM00180582). We map zip codes to counties and merge with the external datasets to get local population density, COVID-19 infection, and death counts. The IRB has also approved this merge (HUM00180640).

\section{Heterogeneity in Actions, Adding Beliefs as Control Variables}

Figure C.1 informs the discussion in Section 4. It plots the estimates without and with beliefs controls (both sets of estimations also include baseline controls and controls for health risk factors and personal experience with COVID-19). Belief controls include respondents' outcome predictions, effectiveness beliefs, and health worries.

Figure C.1: How Actions Vary with Demographics, Controlling for Beliefs, Survey Data

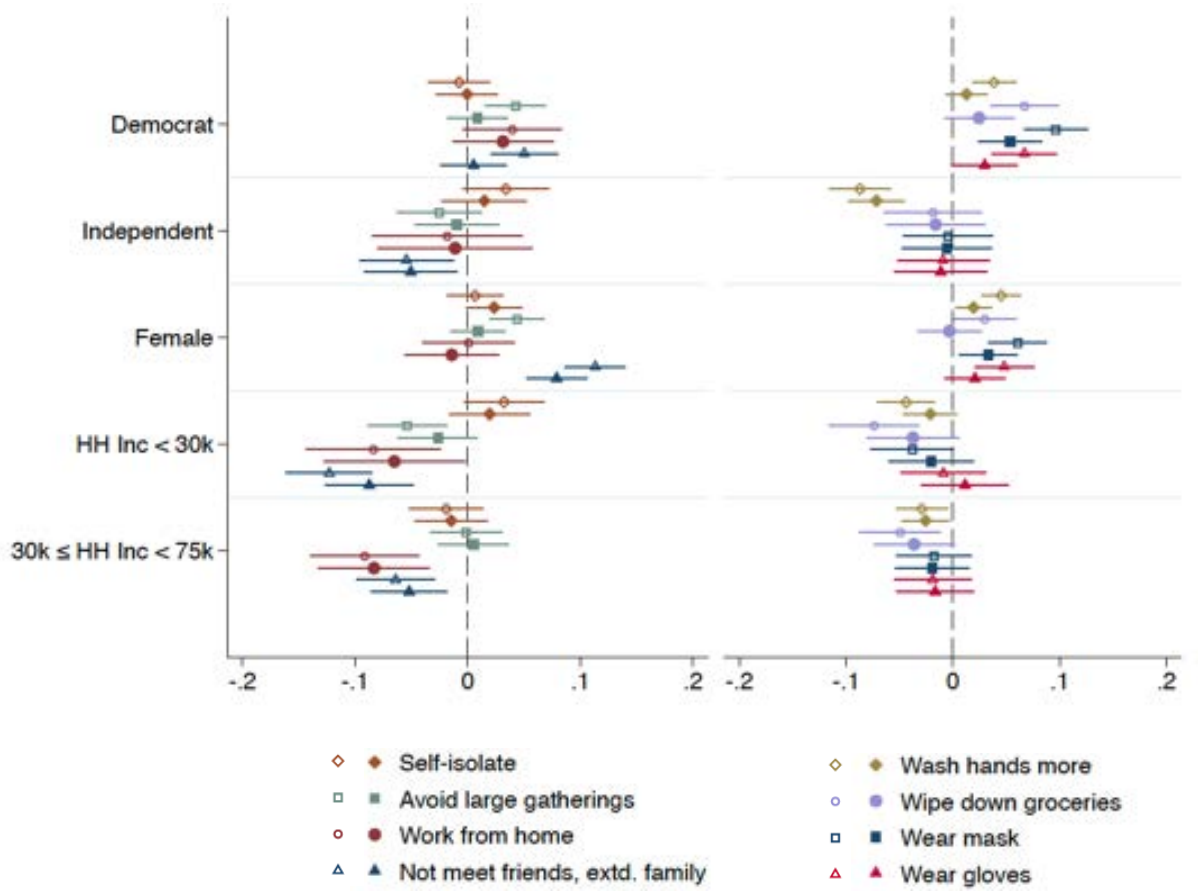

Notes: For each of the actions indicated in the legend, this figure plots two sets of the estimated coefficients $\alpha$ in equation (2) and the corresponding 95\% confidence intervals. The hollow and solid indicators are, respectively, the estimates without and with beliefs controls. Both sets of estimations include baseline controls and controls for health risk factors and personal experience with COVID-19. 


\section{Heterogeneity in Beliefs, Controlling for Information Sources}

Figure D.1 also informs the discussion in Section 4. It plots the estimates without and with news consumption controls (both sets of estimations also include baseline controls and controls for health risk factors and personal experience with COVID-19). News consumption controls include dummy variables for news sources that respondents follow (ABC News, CNN, Fox News Channel, Local news, MSNBC, NPR, HuffPost, The New York Times, The Wall Street Journal, The Washington Post, other, or none) and metrics that capture how much attention they pay to other information sources (friends, family members, pastor and/or spiritual community, the President and his administration, the respondent's governor, scientists/researchers, Centers for Disease Control, people they follow on Twitter, and people they follow on Facebook) rated on a 1-5 scale (5-point rating: not at all--very much so).

Figure D.1: How Beliefs Vary with Demographics, Controlling for Information Sources, Survey Data

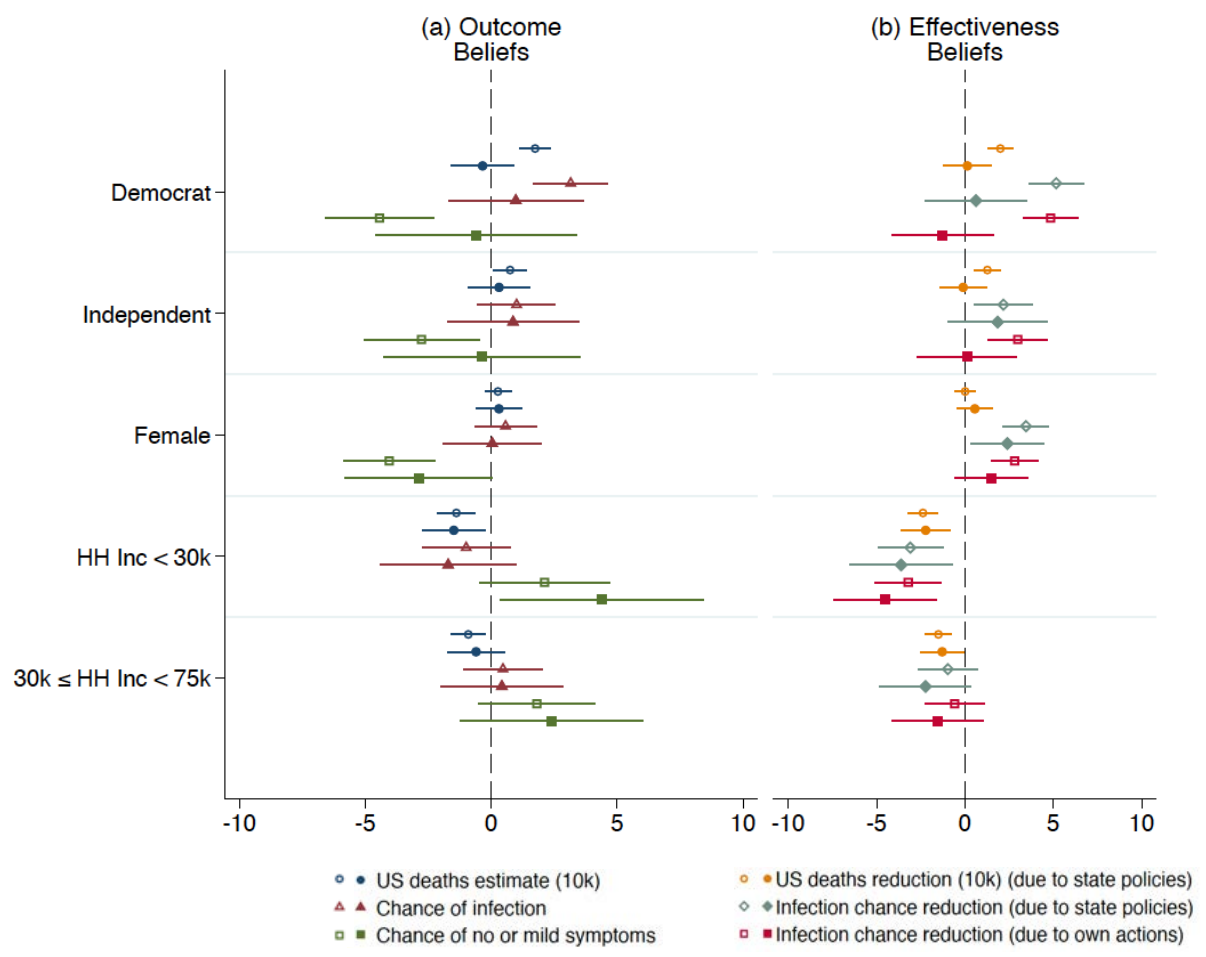

Notes: For each of the beliefs indicated in the legend, this figure plots two sets of the estimated coefficients $\alpha$ in equation (2) and the corresponding 95\% confidence intervals. The hollow and solid indicators are, respectively, the estimates without and with news consumption controls. Both sets of estimations include baseline controls and controls for health risk factors and personal experience with COVID-19. 


\section{SA Robustness: SafeGraph Data Analyses}

We conduct two sets of robustness analyses to show how our results in Section 2 vary with different adjustment methods and control variables.

\section{SA.1 Robustness of Mobility Results w.r.t. Different Controls}

First, we investigate how our controls modify the results. In Figure SA.1, we plot results from three different specifications: (1) a specification with only state-date and county fixed effects as controls; (2) a specification adding socio-economic and demographic controls, i.e., $Z_{c}=Z_{1 c}$, which includes the population shares of whites, blacks and Asians, percent of adults with a bachelor's degree, percent of adults without a high-school degree, unemployment rate, and the employed share of county population; and (3) our baseline specification with the full set of controls, i.e., $Z_{c t}=$ $\left(Z_{1 c}, Z_{2 c t}\right)$, where $Z_{2 c t}$ captures the time-varying differences in COVID-19 risks across counties: logarithm of one plus the number of COVID-19 cases in county $c$ at time $t$, logarithm of one plus the number of COVID-19 deaths, logarithm of population density, population share over the age of 65, degree of urbanization, and a binary variable indicating whether the county is in a metropolitan area.

Figure SA.1 shows that mobility response gaps in leaving home would be larger if we did not include the full set of controls $Z_{c t}$ in our analyses. The gaps are attenuated by the inclusion of demographic controls, and further attenuated by the inclusion of local COVID-19 risks. These results suggest that part of the differences reported by the popular press across these demographic dimensions may be due to other co-varying factors.

\section{SA.2 Robustness of Mobility Results w.r.t. Different Adjustment Methods}

In the SafeGraph data, the number of completely-at-home devices could be under-reported due to a sampling bias. SafeGraph (2020) reports: "GPS data from smartphones is often subject to a sampling bias in favor of devices that are changing locations (i.e., moving). Collecting GPS data is battery intensive, and software applications sometimes implement GPS data collection methods that depend on movement of the device, rather than a fixed time interval. This represents a sampling bias in favor of detecting devices that are moving." 
Figure SA.1: Shares of Devices Engaging in Different Types of Activity, with Different Sets of Controls, SafeGraph Data

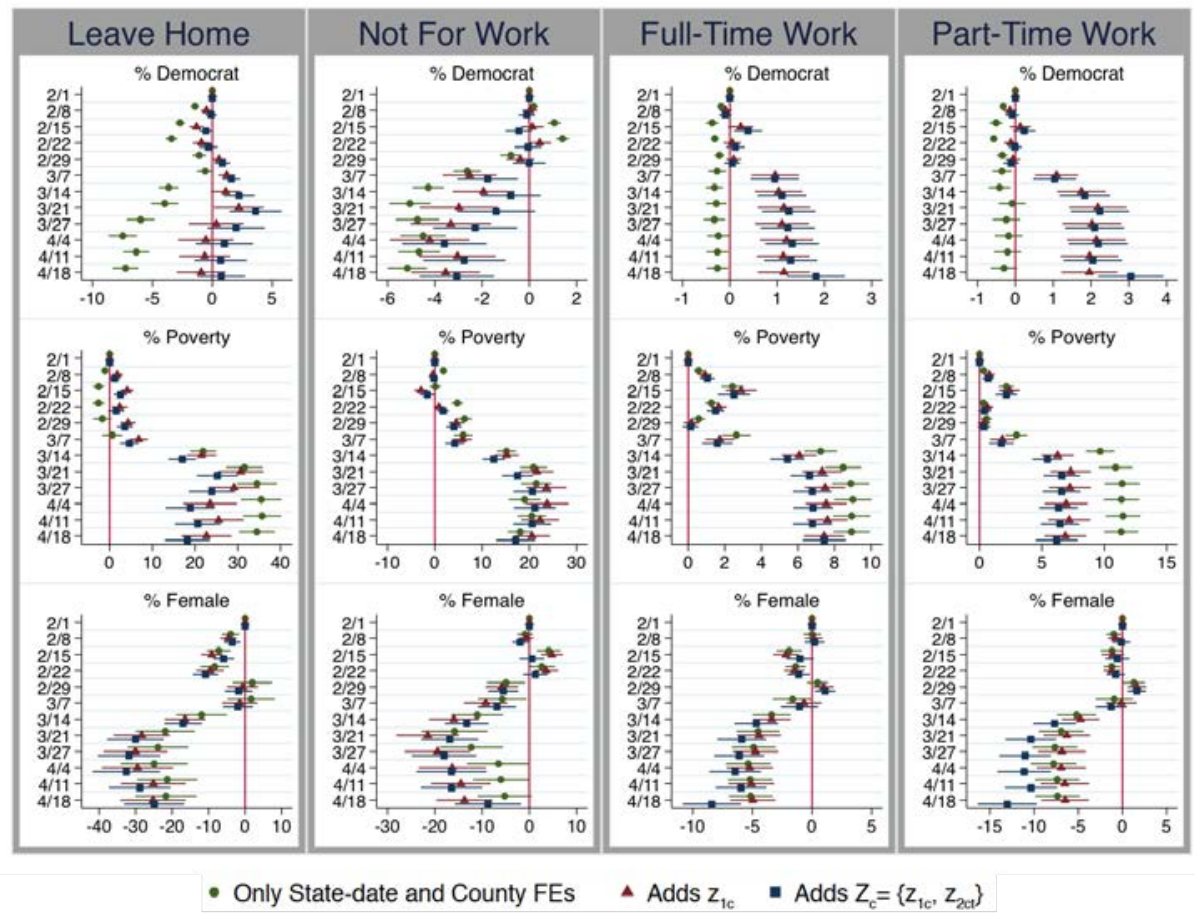

Notes: This figure plots three sets of $\beta_{w(t)}$ estimates and the corresponding $95 \%$ confidence intervals obtained by estimating equation (1) with only state-date and county fixed effects (estimates indicated by green dots), with controls $Z_{1 c}$ added (red triangles) and the full specification with all controls $Z_{1 c}, Z_{2 c}$ (blue squares). Each row plots the estimated coefficient for a specific demographic variable (\% Democrat, \% population below poverty line, and $\%$ female). The y-axis markers indicate the beginning date of the week for which the coefficients are reported. 
In the main text, we report results after adjusting the share of devices at home by designating all inactive devices as completely-at-home. We can do so because SafeGraph reports all devices in its sample during a month, regardless of whether it saw any activity for them on a specific day within the month (candidate device count). This adjustment provides an upper bound of the number of completely-at-home devices. SafeGraph also reports the number of devices that "pinged" during a given day (active device count). In this section, we report results using different metrics and adjustment methods based on candidate device count or active device count. We explore the robustness of our results to the following four alternatives:

1. Not adjusting the share of devices at home. This approach assumes there is no under-reporting of completely-at-home devices. Therefore, the unadjusted share $s_{\text {at-home }}=$ $100 \times($ completely-at-home devices/candidate devices $)$ is a lower bound. This robustness check impacts only the results on the share of people who "Leave Home"; it does not impact the other shares because the denominator for all shares remains the same as in the baseline.

\section{Calculating the number of "expected" active devices based on the maximum ratio} of active devices to candidate devices in the first week. In this approach, we calculate the maximum ratio active devices to candidate devices in a day during the first week, assume it to be the ratio of the latent true number of active devices to candidate devices and assume this ratio to be stable over time. We then calculate the "expected active device count" as the candidate device count multiplied by the initial ratio on each day. We use this expected number of active devices in each county as the base and adjust the at-home device count accordingly. We define the dependent variables as: $s_{\text {part-time }}=100 \times\left(\frac{\text { part-time work devices }}{\text { expected device count }}\right)$, $s_{\text {full-time }}=100 \times\left(\frac{\text { full-time work devices }}{\text { expected device count }}\right), s_{\text {leave-home }}=100-s_{\text {at-home }}$, where $s_{\text {at-home }}=100 \times$ $\left(\frac{\text { completely-at-home devices }+\max \{0 \text {,expected device count - active devices }\}}{\text { expected device count }}\right)$, and $s_{\text {not-work }}=100-s_{\text {at-home }}-$ $s_{\text {part-time }}-s_{\text {full-time }}$.

3. Using active devices as the base for computing shares, and not adjusting for under-reporting. This approach provides an alternative lower bound for the share of athome devices. The dependent variables are defined as: $s_{\text {part-time }}=100 \times\left(\frac{\text { part-time work devices }}{\text { active devices }}\right)$, $s_{\text {full-time }}=100 \times\left(\frac{\text { full-time work devices }}{\text { active devices }}\right), s_{\text {leave-home }}=100-s_{\text {at-home }}$ where $s_{\text {at-home }}=100 \times$ $\left(\frac{\text { completely-at-home devices }}{\text { active device }}\right)$, and $s_{\text {not-work }}=100-s_{\text {at-home }}-s_{\text {part-time }}-s_{\text {full-time }}$. 
4. Using the maximum active devices in the first week for each county as the base and adjusting the at-home devices accordingly. In this approach, we calculate the largest number of active devices in a day during the first week for each county ("initial active device"), assume it to be the latent true number of active devices and assume this latent number to be stable over time. This approach provides an alternative upper bound for the share of at-home devices. We define the dependent variables as: $s_{\text {part-time }}=100 \times$ $\left(\frac{\text { part-time work devices }}{\text { initial active devices }}\right), s_{\text {full-time }}=100 \times\left(\frac{\text { full-time work devices }}{\text { initial active devices }}\right), s_{\text {leave-home }}=100-s_{\text {at-home }}$, where $s_{\text {at-home }}=100 \times\left(\frac{\text { completely-at-home devices }+\max \{0, \text { initial active devices - active devices }\}}{\text { initial active devices }}\right)$, and $s_{\text {not-work }}=$ $100-s_{\text {at-home }}-s_{\text {part-time }}-s_{\text {full-time }}$

In Figure SA.2, we report estimates from these robustness specifications. The first column, where the dependent variable is 'Leave Home,' depicts results from all four specifications. Other columns report results from the latter three specifications, since the first alternative does not impact the construction of these three dependent variables. Although the results change with the assumptions, the general message of our findings stays the same. 
Figure SA.2: Mobility Differences, SafeGraph Data, Robustness Checks

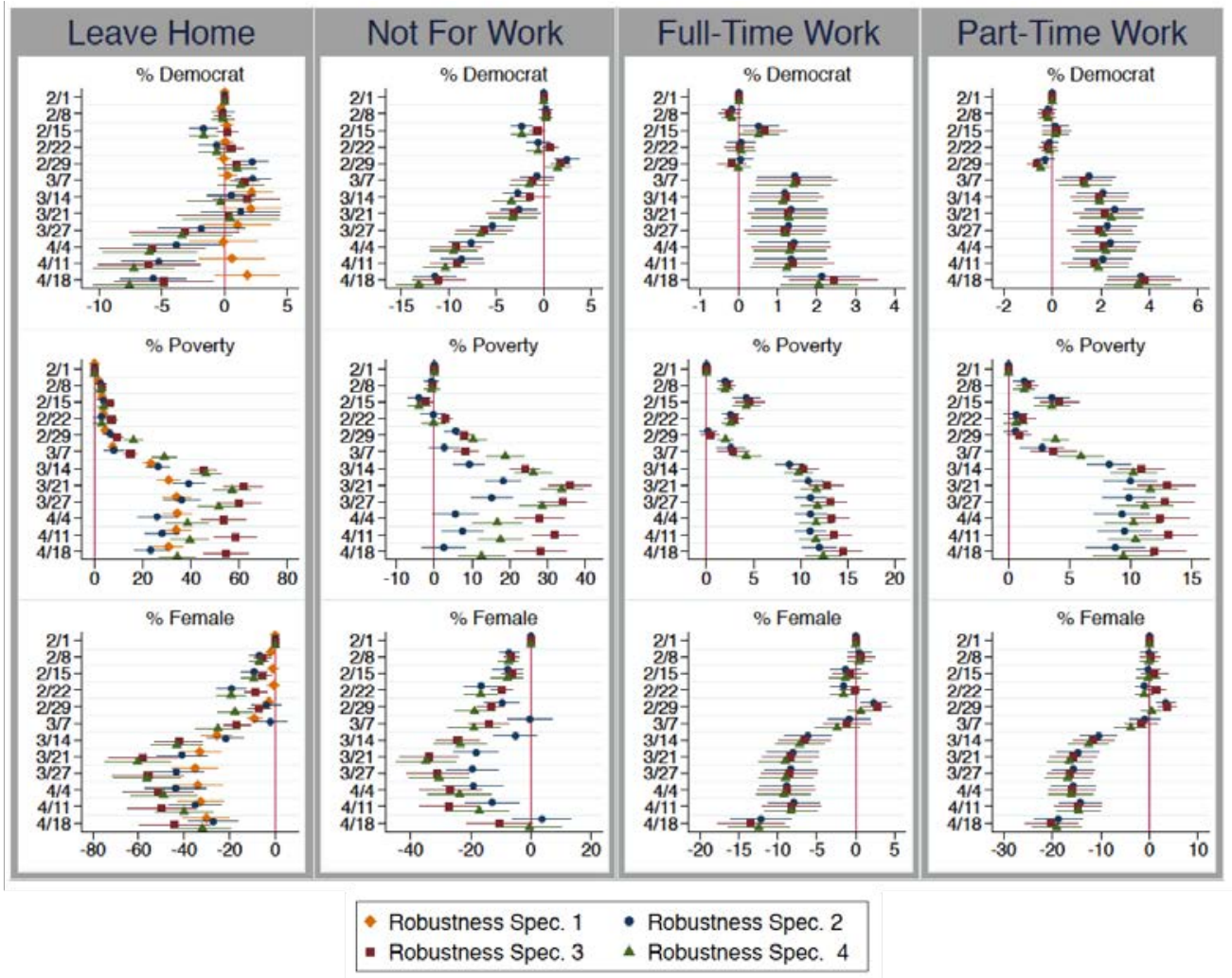

Notes: This figure plots four sets of $\beta_{w(t)}$ estimates and the corresponding $95 \%$ confidence intervals obtained by estimating equation (1) with each alternative robustness specification outlined in Appendix SA.2. Each row plots the estimated coefficient for a specific demographic variable (\% Democrat, $\%$ population below poverty line, and $\%$ female). The y-axis markers indicate the beginning date of the week for which the coefficients are reported. 


\section{SB Robustness: Survey Data Analyses}

In this section, we present robustness checks of our survey results. Just as gender, party affiliation, and income may co-vary with other demographics, they may also co-vary with health risks and personal experience with COVID-19, which in turn may impact individuals' actions and beliefs. Therefore, we collected data on these factors and checked the robustness of our results by including them as controls. Figures SB.1 and SB.2, on action heterogeneity and belief heterogeneity, respectively, report results from a specification including controls for personal health risks and personal experience with the disease. Personal experience variables include whether the respondent had the disease, whether she/he had a family member or a close friend who got ill with COVID19, and whether she/he has a family member or a close friend who was hospitalized or died due to COVID-19. The question on personal health risks is based on the risk factors listed by the Centers for Disease Control on April $1^{\text {st }}$, including moderate to severe asthma; COPD or other chronic lung disease; serious heart conditions; diabetes; conditions that can cause a person to be immunocompromised, including cancer treatment, smoking, bone marrow or organ transplantation, immune deficiencies, poorly controlled HIV or AIDS, and prolonged use of corticosteroids and other immune-weakening medications; severe obesity (BMI of 40 or higher); chronic kidney disease and currently undergoing dialysis; and liver disease. Because Wave 1 surveys did not ask the personal health risk question, we also replicate our main results excluding Wave 1 respondents as a baseline for comparison in the figure (coefficients indicated with hollow markers). Including health risks and personal experience controls does not substantially change our results. 
Figure SB.1: How Actions Vary with Demographics, Controlling for Health Risks and COVID-19 Experience, Survey Data

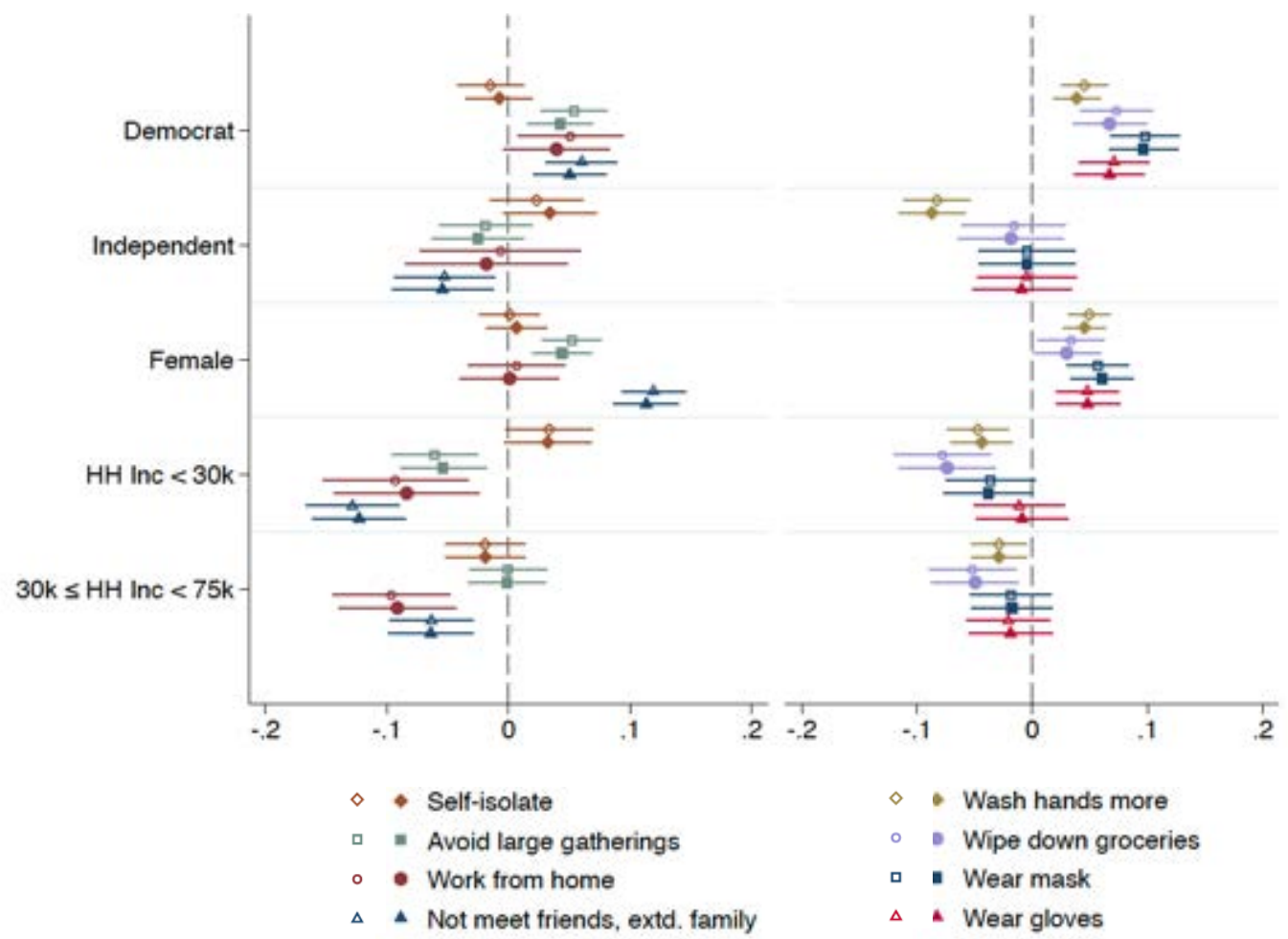

Notes: For each action indicated in the legend, this figure plots two sets of the estimated coefficients $\alpha$ in equation (2) and the corresponding 95\% confidence intervals. The hollow and solid indicators are the estimates from specifications without and with controls for individuals' health risks and personal experience with COVID-19. Both sets of estimations exclude data from Wave 1. 
Figure SB.2: How Beliefs and Worries Vary with Demographics, Controlling for Health Risks and COVID-19 Experience, Survey Data

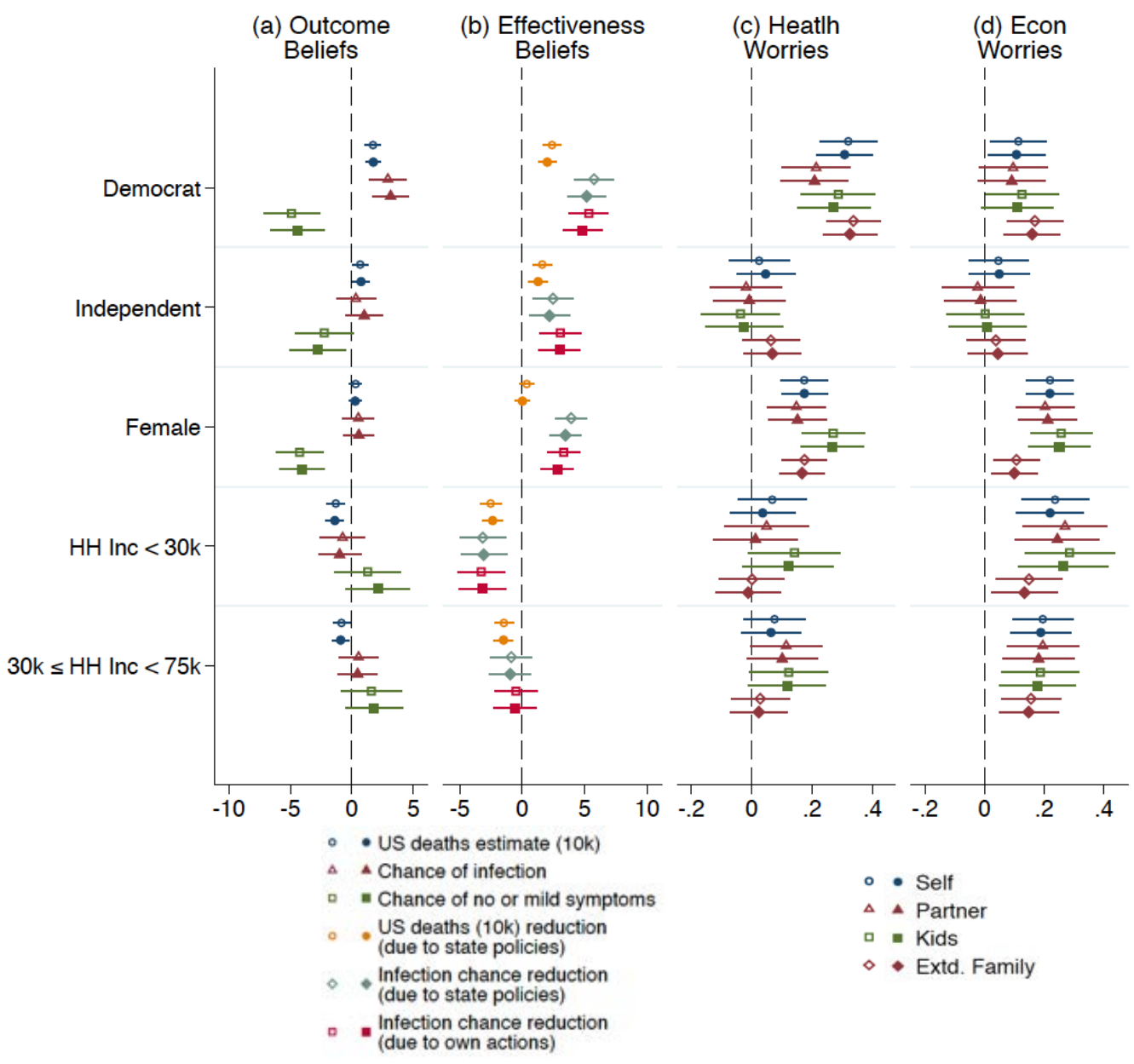

Notes: For each of the predictions and worries indicated in the legend, this figure plots two sets of the estimated coefficients $\alpha$ in equation (2) and the corresponding 95\% confidence intervals. The hollow and solid indicators are the estimates from specifications without and with controls for individuals' health risks and personal experience with COVID-19. Both sets of estimations exclude data from Wave 1. In panels (a) and (b), the dependent variables are elicited predictions indicated in the legend. In panels (c) and (d), the dependent variables are the extent to which respondents are worried about the well-being of the groups indicated in the legend.

\section{SC Survey Representativeness, Consent, Questions}

\section{SC.1 Representativeness of Survey Data}

Lucid collects granular demographic data from respondents on age, education, household income, political affiliation, gender, ethnicity, and region, while providing respondent anonymity to researchers.

Lucid promises national representation based on age, gender, ethnicity, and regions of the U.S. 
Table SC.1: Summary Statistics on Demographics of Survey Respondents

\begin{tabular}{lcc}
\hline & Mean & National Mean $^{a}$ \\
\hline \hline Age: $18-44$ & 0.49 & $0.48^{b}$ \\
Age: $65+$ & 0.17 & $0.17^{b}$ \\
Asian & 0.05 & 0.06 \\
Black & 0.10 & 0.13 \\
White & 0.75 & 0.76 \\
Female & 0.52 & 0.51 \\
Region - Midwest & 0.19 & 0.21 \\
Region - Northeast & 0.20 & 0.17 \\
Region - South & 0.38 & 0.38 \\
Region - West & 0.22 & 0.24 \\
\hline Democrat & 0.39 & 0.31 \\
Republican & 0.33 & 0.27 \\
High school graduate or higher & 0.97 & $0.88^{c}$ \\
Bachelor's degree or higher & 0.45 & $0.32^{c}$ \\
Household income $<30 k$ & 0.32 & 0.23 \\
Household income $\in[30 k, 75 k)$ & 0.37 & 0.34 \\
\hline${ }^{a}$ Data source: Census except for political affiliation, which is from Gallup for April 1 - 14, 2020. \\
See https://news.gallup.com/poll/15370/party-affiliation.aspx. \\
${ }^{b}$ Share of persons age 18+ years. & & \\
${ }^{c}$ Share of persons age 25+ years. & &
\end{tabular}

We can also confirm the representation of our sample. Table SC.1 provides the breakdown of demographic variables among our respondents. The top panel confirms that our sample indeed tracks well with the U.S. national benchmarks in age, ethnicity, gender, and regions of the U.S. Our survey over-samples Democrats, more-educated people and lower-household-income people.

\section{SC.2 Survey Consent}

The survey starts with the following consent form:

You are invited to participate in a research study about COVID-19. This is a 15-minute long survey that will ask about your perceptions, expectations and feelings about the disease, its effects on you and on our nation. If you agree to be part of the research study, you will be asked to provide your opinions on policies, risks, and will be asked to answer questions related to your current situation. Please pay attention to all questions. We will include several attention checks.

Benefits of the research to the public stem from your participation and honest answers. Using this survey data, we hope to be able to provide guidelines for assessing and responding to differences 
across communities. Risks and discomforts: Thinking about COVID-19 and its impact may induce negative emotions, like anxiety or fear. These risks and discomforts are minimal for most people.

Participating in this study is completely voluntary. Even if you decide to participate now, you may change your mind and stop at any time. You may choose not to continue with the survey at any time and for any reason.

There is no deception or false information in this survey.

We will protect the confidentiality of your research records by not publishing any information that may identify you. Information collected in this project may be shared with other researchers, and may be connected to other aggregate datasets at the county level. We will not share any information that could identify you. All results will be reported in aggregate.

Principal Investigator: Yesim Orhun, Associate Professor, University of Michigan. If you have questions about this research study, please contact Prof. Yesim Orhun by emailing aorhun@umich.edu. The University of Michigan Institutional Review Board Health Sciences and Behavioral Sciences has determined that this study is exempt from IRB oversight.

By clicking to proceed, you are confirming that you read this page and are providing consent to participate.

\section{SC.3 Survey Questions}

In what follows, survey questions are in normal font while our notes are in italic.

\section{Risk tolerance}

Thinking about yourself, in general, how willing or unwilling are you to take risks? Please use the scale below, ranging from 0 to 10, where 0 means "completely unwilling to take risks" and a 10 means you are "very willing to take risks." You can also use any number between 0 and 10 to indicate where you fall on the scale. [Scale: 1 to 10, choose one.]

\section{Employment and Economic Impact, Zip code}

- What's your current employment status? [Choose one: Employed full time; Employed part time; Furloughed; Unemployed (before the coronavirus); Unemployed (after the coronavirus); Retired; Student; Prefer not to answer.] 
- Please think of everyone in your household who was earning an income before the coronavirus crisis. What was the economic impact of the coronavirus situation on the income of your household? [Choose one: Greatly negative; Very negative; Somewhat negative; No change; Somewhat positive; Very positive; Greatly positive.]

- What is your zip code? [Fill in.]

\section{Personal Experience with COVID-19}

- How has the health of the community you live in been impacted by the coronavirus? [Choose one: So far, we don't have any cases or deaths; We have only a few cases and no deaths; We have a moderate number of cases, but no deaths; We have a moderate number of cases, and at least one death; We have a lot of cases, but no deaths; We have a lot of cases, and at least one death; We have a lot of cases and a lot of deaths.]

- Have you been infected with the coronavirus? [Choose one: Yes, I tested positive; No, I tested negative; Probably yes, but I did not get tested; Probably not, but I did not get tested.]

- Do you have friends or family members who have been severely ill with the coronavirus? [Choose one: Yes; We suspect it was due to coronavirus, but we don't know for sure; No.]

- Do you have friends or family members who have been hospitalized or have died due a coronavirus infection? [Choose one: Yes; We suspect it was due to coronavirus, but we don't know for sure; No.]

- Do you have any health conditions you know of that put you in the high-risk category for serious complications arising from a coronavirus infection? [Choose one: Definitely yes; Most likely yes; I am not sure; Most likely not; Definitely not.]

\section{Attention check, screening}

- Please think of everyone in your community who has been affected by the coronavirus crisis. It is important that you pay attention to this survey. Please check greatly positive below. [Choose one: Greatly negative; Very negative; Somewhat negative; No change; Somewhat positive; Very positive; Greatly positive.] Those who failed this attention check were not allowed to proceed with the rest of the survey and their survey responses were not recorded. 


\section{State Restrictions and Own Precautions}

- Has your state introduced any social distancing measures? Please click all that apply in your state at this time. [Choose all that apply: No social distancing measures at this time; Schools are closed; Congregating at churches is not allowed; Restaurants/bars closed, except for takeout/delivery; Gyms are closed; Workers cannot travel to work at non-essential businesses; Gatherings of $50+$ people are forbidden, less than 50 is ok; Gatherings of $10+$ people are forbidden, less than 10 is ok; All social gatherings are forbidden; Stay-at-home order.]

- Which of the following changes have you made to protect yourself from the coronavirus infection? Please click all that apply. [Choose all that apply: I did not make any changes; Wash hands more frequently; Canceled travel plans; Avoid large gatherings; Work from home; Wear gloves when I go shopping; Wipe down groceries after I bring them home; Wear a mask when I am out and about; Do not meet any of my friends or extended family in-person; Avoid all public places and self-isolate at home; I made other changes: (fill in).]

\section{Worries}

- How worried are you feeling for the health of the following people? If you don't have the people mentioned in some statements (partner, kids, extended family), please click "Not Applicable". [Groups of people: My own health; My partner's health; My kids' health; My extended family's health; The health of doctors and nurses in my community; The health of other members of my community; The health of people in big cities like New York, Seattle, Detroit, San Francisco; The health of all the people in the US.] [Choose one: Not at all worried, slightly worries, moderately worried, very worried, extremely worries. Also option: not applicable.]

- How worried are you feeling for the economic well being of the following people? [Same options and groups as above.]

\section{Beliefs About Infection Risk}

As of April 20, 2020, CDC (Centers for Disease Control and Prevention) is reporting 776,093 confirmed coronavirus cases and 41,758 deaths in the U.S. Many cases go undetected. ${ }^{11}$ Of course,

\footnotetext{
${ }^{11}$ The date and these two numbers were updated to the applicable information of two days prior to the survey time.
} 
infection rates depend on the community and the protection measures each person can take. Assuming that the social distancing policies and your personal efforts stay the same, what are the chances that you will get infected with the coronavirus in the next three months? [Choose one: $0 \%$ chance; $1-10 \%$ chance; $11-20 \%$ chance; $21-30 \%$ chance; $31-40 \%$ chance; $41-50 \%$ chance; $51-60 \%$ chance; $61-70 \%$ chance; $71-80 \%$ chance; $81-90 \%$ chance; $91-100 \%$ chance.]

\section{Beliefs About the Effectiveness of State Restrictions and Own Precautions in Reducing Infection Risk}

The next two questions embedded responses from previous questions. If respondent indicated that their state has not introduced any social distancing measures, or that they have not taken any precautions, the relevant question was not displayed to the respondent.

- Earlier, the survey asked about the changes you personally made to protect yourself from the coronavirus infection. You indicated that you [all precautions the the respondent indicated as having taken]. Assuming that the state policies stay the same, what do you think your chances of becoming infected with coronavirus in the next three months would be if you did not make these changes? [Choose one: Same chance; $5 \%$ higher chance; $10 \%$ higher chance; $15 \%$ higher chance; $20 \%$ higher chance; $25 \%$ higher chance; $30 \%$ higher chance; $40 \%$ higher chance; $50 \%$ higher chance; My chance of being infected would increase by more than $50 \%$.]

- Imagine that you could still take the same measures you personally took to lower your chances of being infected with coronavirus, but your state had not introduced any social distancing measures. Assuming your personal efforts stay the same, what do you think your chances of becoming infected with coronavirus in the next three months would be if your state did not have social distancing measures? [Choose one: Same chance; $5 \%$ higher chance; $10 \%$ higher chance; $15 \%$ higher chance; $20 \%$ higher chance; $25 \%$ higher chance; $30 \%$ higher chance; $40 \%$ higher chance; $50 \%$ higher chance; My chance of being infected would increase by more than $50 \%$.]

\section{Beliefs About Health Outcomes Conditional on Being Infected}

- According to the CDC (Centers for Disease Control and Prevention) report, about $7 \%$ of people diagnosed with the coronavirus are hospitalized, but do not need intensive care. About 
$1.5 \%$ of people are hospitalized and need intensive care. It is also suspected that a large percentage of people are symptom free and/or have mild versions of the disease.

Most importantly, the chances are person-specific. The progression of the disease can be very different based on your age, health, pre-existing condition, living conditions, how much of the virus you are exposed to, etc. Although it's hard to know without data, you probably have a better understanding of your situation than anyone else. Therefore, we ask you to predict how the coronavirus is likely to affect you, should you get infected:

Please make sure numbers add up to 100. Allocate points according to how big you think your chances are for each possibility. [Chances that I will be symptom free are: (fill in, numerical); Chances that I will have a mild version of the disease are: (fill in, numerical); Chances that I will have a moderate version (without hospitalization) are: (fill in, numerical); Chances that I will have a severe version that requires hospitalization (but no further interventions) are: (fill in, numerical); Chances that I will have a severe version that requires intensive care at the hospital are: (fill in, numerical).]

\section{Factual Questions About Factors That Influence Health Outcomes}

- Quality of care. Communities differ in how well equipped their hospitals are and how much capacity their hospitals have when it comes to coronavirus infections. If you were to be hospitalized due to coronavirus, what's your expectation of the quality of care you would receive? [Choose one: Top-notch care, no issues; Good care, as usual; Good care, but hospital would have bed/equipment shortages; OK care, as usual; OK care, but hospital would have bed/equipment shortages; Not so good care, as usual; Not so good care, and hospital would have bed/equipment shortages.]

- Pre-existing health conditions. The CDC (Centers for Disease Control and Prevention) released the list of underlying medical conditions that put people of all ages at higher risk for severe illness resulting from the coronavirus infection. We list them below.

Which of these apply to you? Please click all that apply. [Choose all that apply: Moderate to severe asthma; COPD or other chronic lung disease; Serious heart conditions; Diabetes; Conditions that can cause a person to be immunocompromised, including cancer treatment, 
smoking, bone marrow or organ transplantation, immune deficiencies, poorly controlled HIV or AIDS, and prolonged use of corticosteroids and other immune weakening medications.; Severe obesity (BMI of 40 or higher); Chronic kidney disease and currently undergoing dialysis; Liver disease; I do not want to answer; None of them apply to me.]

\section{Beliefs About Systemic Health Risk (Total Number of Deaths in the U.S.)}

- As of April 20, 2020, CDC (Centers for Disease Control and Prevention) is reporting 41,758 deaths in the US. ${ }^{12}$ Assuming the state policies remain the same, how many people do you think will die from a coronavirus infection in the U.S. by July 1, 2020? [Choose one: less than 25,$000 ; 25,000-50,000 ; 50,000-75,000 ; 75,000-100,000 ; 100,000-125,000 ; 125,000-150,000$; 150,000-175,000; 175,000-200,000; 200,000-225,000; 225,000-250,000; 250,000-275,000; 275,000-300,000; 300,000-350,000; more than 350,000.]

\section{Beliefs About Economic Outcomes}

- The U.S. gross domestic product (GDP) grew about 2.3\% in 2019. Assuming the state policies remain the same, how much GDP growth do you expect in 2020? [Choose one: more than 10\% growth; $5 \%-10 \%$ growth; $2.5 \%-5 \%$ growth; $0-2.5 \%$ growth; $0 \%$ to $-2.5 \%$ growth (negative growth means contraction); $-2.5 \%$ to $-5 \%$ growth (negative growth means contraction); $-5 \%$ to $-10 \%$ growth (negative growth means contraction); $-10 \%$ to $-20 \%$ growth (negative growth means contraction); $-20 \%$ to $-30 \%$ growth (negative growth means contraction); worse than $-30 \%$ growth (negative growth means contraction).]

- In the last quarter of 2019, unemployment rate in the U.S. was 3.6\%. Assuming the state policies remain the same, how much unemployment do you expect in the U.S. by July 1, 2020? [Choose one: less than 3\%; 3-5\%; 5-10\%; 10-15\%; 15-20\%; 20-25\%; 25-30\%; more than 30\%.]

Beliefs About the Effectiveness of State Policies in Reducing Total Number of Deaths in the U.S. and Their Impact on Economic Outcomes

\footnotetext{
${ }^{12}$ The date and the number of deaths were updated to the applicable information of two days prior to the survey time.
} 
At this time, the majority of states are implementing some measure of social distancing. Assuming there are no changes to these policies, you expect that by July 1st [reminder of their previous death prediction] people in the U.S. will die due to coronavirus infections. You also expect [reminder of their previous unemployment prediction] rate of unemployment by July 1st, and [reminder of their previous GPD growth prediction] in GDP in 2020. How would these predictions change if none of the states implemented any social distancing measures?

- Please select the number of U.S. deaths you would expect by July 1st if there were no social distancing measures. [Choose one: less than 25,000; 25,000-50,000; 50,000-75,000; 75,000100,000; 100,000-125,000; 125,000-150,000; 150,000-175,000; 175,000-200,000; 200,000-225,000; $225,000-250,000 ; 250,000-275,000 ; 275,000-300,000 ; 300,000-350,000$; more than 350,000 .]

- Please select the rate of GDP growth you would expect in 2020 if there were no social distancing measures. [Choose one: more than $10 \%$ growth; $5 \%-10 \%$ growth; $2.5 \%-5 \%$ growth; 0-2.5\% growth; $0 \%$ to $-2.5 \%$ growth (negative growth means contraction); $-2.5 \%$ to $-5 \%$ growth (negative growth means contraction); $-5 \%$ to $-10 \%$ growth (negative growth means contraction); $-10 \%$ to $-20 \%$ growth (negative growth means contraction); $-20 \%$ to $-30 \%$ growth (negative growth means contraction); worse than $-30 \%$ growth (negative growth means contraction).]

- Please select the rate of unemployment you would expect by July 1st if there were no social distancing measures. [Choose one: less than 3\%; 3-5\%; 5-10\%; 10-15\%; 15-20\%; 20-25\%; 25-30\%; more than 30\%.]

\section{Attitudes Towards Policies}

- Policy makers are debating the effectiveness and pros/cons of many options. We listed some of these options below. Which of them do you most agree/disagree with regarding their sensibility at this time, in your community? [Policy recommendations: Washing hands more often, avoiding handshakes; Keeping kids out of school; Not sending people to work, unless they are essential workers; Forbidding all social gatherings; Requiring people to stay at home.] [Choose one: Strongly disagree; Disagree; Somewhat disagree; Neither agree nor disagree; Somewhat agree; Agree; Strongly agree.] 


\section{Impact of the pandemic on the respondent, general}

- Please rate how much trouble you are currently having with the following issues: [Issues: Having enough money for food and housing (rent, mortgage payments); Having enough money for medications; Having enough money for other expenses and/or savings; Finding groceries, even if you have the money to buy them; Finding medications, even if you have the money to buy them; Maintaining a positive outlook and not getting anxious; Mental health overall.] [Choose one: No trouble at all; Small problems; Some difficulties; Many difficulties; Serious trouble.]

\section{Constraints as Main Drivers of Choices}

Everyone has different preferences, risks, constraints in their lives. Earlier in the survey, we asked you what self-protective measures you took, if any. On this page, we ask why you did not choose some of the self-protective actions. These questions are not meant as a judgement. They aim to understand what actually drives people's decisions. Please answer them honestly. We want to know more about people's preferences and circumstances. The questions below were asked if the applicable precaution was not taken by the respondent.

- You indicated that working from home was not one of the changes you made. Which of the following best describes why? [Choose one: I am retired/a student/currently not working; I could perhaps work from home, but it's better not to/I don't like to; I was not given the option to work from home (essential worker or employer needed me); I cannot work from home; Other: (fill in).]

- You indicated that wearing gloves when you go shopping was not one of the changes you made. Which of the following best describes why? [Choose one: It's not necessary; I would like to, but cannot find gloves; I don't shop for anything, including groceries; Other: (fill in).]

- You indicated that wearing masks was not one of the changes you made. Which of the following best describes why? [Choose one: Masks are not useful; I do not like wearing masks; I would like to, but cannot find or afford masks; I do not leave my house even to take a walk; Other: (fill in).] 
- You indicated that avoiding all public places and self-isolating was not one of the changes you made. Which of the following best describes why? [Choose one: It's too much. We need to keep functioning; I would like to, but I have to work outside the home; I would like to, but I have to leave my house regularly for doctor visits; I would like to, but I have to go get food and groceries - I cannot afford to have it delivered; I would like to, but I have to go get food and groceries - I don't want to have it delivered; I would like to, but I have to go get food and groceries — there are no deliveries available; Other: (fill in).]

- You indicated that canceling travel plans was not one of the changes you made. Which of the following best describes why? [Choose one: I did not have any travel plans to begin with; I really wanted to go on the trip/did not feel like canceling; I could not get a refund and did not want to waste the money; I had to travel because of work obligations; I traveled because of family obligations; other: (fill in).]

- You indicated that avoiding large gatherings was not one of the changes you made. Which of the following best describes why? [Choose one: It's too much. We need to keep functioning; I would like to, but I cannot avoid large gatherings because of my work; Other: (fill in).]

\section{News sources}

- Which news sources do you usually rely on? [Choose all that apply: ABC News; CNN; Fox News Channel; Local news; NBC / MSNBC; NPR (Public Radio); Huff Post; The New York Times; The Wall Street Journal; Washington Post; Other: (fill in); I don't follow any news.]

- What other sources of information do you mostly pay attention to? [Other sources: My friends; My family members; My pastor and/or our spiritual community; The President and his administration; Our Governor; Scientists/researchers; CDC (Center for Disease Control); People I follow on Twitter; People I follow on Facebook.] [Choose one: Not at all; A little bit; Somewhat; Mostly; Very much so; Not applicable.]

The study ended with inviting comments, if respondents had any, about the survey. 